\title{
Genetic based optimization for multicast routing algorithm for MANET
}

\author{
$\mathrm{C} \mathrm{RAJAN}^{1, *}$ and $\mathrm{N} \mathrm{SHANTHI}^{2}$ \\ ${ }^{1}$ K. S. Rangasamy College of Technology, Tiruchengode, Tamilnadu 637215, India \\ ${ }^{2}$ Nandha Engineering College, Erode, Tamilnadu, 638052, India \\ e-mail: csg.rajan12@gmail.com
}

MS received 21 November 2013; revised 6 July 2015; accepted 30 August 2015

\begin{abstract}
Mobile Ad hoc Network (MANET) is established for a limited period, for special extemporaneous services related to mobile applications. This ad hoc network is set up for a limited period, in environments that change with the application. While in Internet the TCP/IP protocol suite supports a wide range of application, in MANETs protocols are tuned to specific customer/application. Multicasting is emerging as a popular communication format where the same packet is sent to multiple nodes in a network. Routing in multicasting involves maintaining routes and finding new node locations in a group and is NP-complete due to the dynamic nature of the network. In this paper, a Hybrid Genetic Based Optimization for Multicast Routing algorithm is proposed. The proposed algorithm uses the best features of Genetic Algorithm (GA) and particle swarm optimization (PSO) to improve the solution. Simulations were conducted by varying number of mobile nodes and results compared with Multicast AODV (MAODV) protocol, PSO based and GA based solution. The proposed optimization improves jitter, end to end delay and Packet Delivery Ratio (PDR) with faster convergence.
\end{abstract}

Keywords. Mobile Ad hoc Network (MANET); multicast routing; MAODV routing; genetic algorithm.

\section{Introduction}

Mobile ad hoc networks (MANETs) are autonomous mobile host systems connected by wireless links, forming a communication network modelled as an arbitrary communication graph without any infrastructure. In MANET the network topology changes dynamically and unpredictably as nodes move freely. Since network bandwidth is scarce multicasting finds effective use in MANET as reduction in transmission overheads improve the Quality of Service (QOS). Multicasting is datagram transmission to a group of hosts identified by one destination address and intended for group-oriented computing (Viswanath et al 2006). Wireless link efficiency is improved when multicasting is used as a single message copy can be used to all nodes that are in

${ }^{*}$ For correspondence 
the transmission range of the sender. The challenges in the design of multicasting system include group member's mobility leading to link breakages, transient loops in the distribution structure, reconfiguration due to mobility which increases channel overhead. To overcome this, various multicast protocols have been proposed in the literature and can be broadly classified as shown in figure 1 .

Tree-based multicast routing protocols are divided into source-rooted and core-rooted schemes based on multicast trees roots. In a source-rooted tree-based multicast routing protocol, source nodes will act as the multicast tree root and process the methodology to distribute tree construction and maintenance. For source rooted multicast routing the source has to know the addresses of all the receivers and topology information of the network. Source-rooted tree protocols have the disadvantage of higher control traffic overhead when used in dynamic networks. In a core-rooted tree multicast routing protocol, nodes which can handle multicast data distribution are selected as cores and it handles the tree construction and maintenance. Tree-based protocols establish minimum paths between the sender and the receivers requiring lesser number of copies to be sent between source and receivers and hence efficiently managing energy among the nodes. However due to the single link between the source and receivers they are more prone to link failures when the nodes are dynamic. Popular tree-based protocols include Ad hoc Multicast Routing protocol using Increasing id-numberS (AMRIS) (Wu \& Tay 1999), Multicast Ad hoc On demand Distance Vector (MAODV) (Kunz \& Cheng 2001), and Ad hoc Multicast Routing Protocol (AMRoute) (Xie et al 2002).

In mesh-based multicast routing protocol, the same packet is distributed among different receivers using a mesh of interconnected nodes. Route discovery is achieved by broadcasting or using core points. As mesh-based protocols provide redundant paths they are relatively more rugged than tree-based protocols when the nodes are mobile. Due to higher control messages they are inefficient in energy savings. Popular techniques include Core Assisted Mesh Protocol (CAMP) (Lee et al 2002) and On Demand Multicast Routing Protocol (ODMRP) (Yi et al 2003). Hybrid-based multicast routing protocols use the best features of mesh-based and treebased routing protocols. However the trees they create are sub-optimal compared to tree-based protocols. Popular techniques include Differential Destination Multicast (DDM) (Ji \& Corson 2001) and Efficient Hybrid Multicast Routing Protocol (EHMRP) (Biswas et al 2004).

Multicast routing protocols are designed to reduce data traffic for packet delivery in a network. When QoS is considered, some protocols can be unsatisfactory based on the network condition. In MANETs QoS is harder to guarantee as bandwidth is shared by all the nodes in the neighborhood and network topology changes due to node mobility. To achieve QOS the nodes have to collaborate to secure resources and establish routes which may not necessarily be the shortest path. Based on the domain where the network will be used appropriate QoS metrics like bandwidth, packet loss rate, delay and cost for multicast routing should be addressed. In multicasting, the routing time will be intractable polynomial time as the same data is sent to multiple destinations (Cheng \& Yang 2010). Optimization of the protocol is required to scale

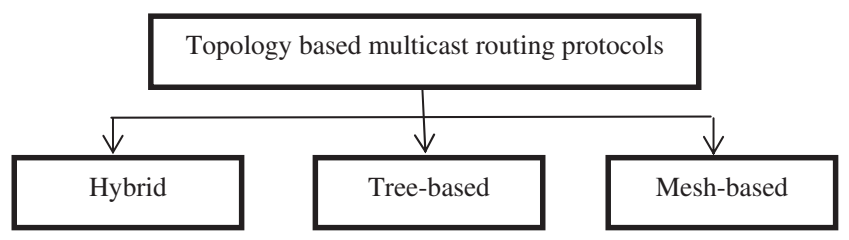

Figure 1. Multicast routing protocol classification. 
the network, reduce costs and use the resources optimally. Therefore, finding multicast tree is a multi-objective optimization problem. The goals of optimization multicast routing are to allocate the available resources of the network in efficient way, to balance the current load in the network, to avoid/reduce the congestion and to provide the required level of QOS to the end users of the network.

Various optimization techniques have been investigated in the literature including techniques to solve the NP complete problem of Multicasting. Zhu \& Jin (2009), Liu et al (2012), Szymaniak et al (2008) and Idboufker et al (2006) used Genetic Algorithm (GA) based heuristics to improve the QoS of multicast network. Zheng et al (2012), Delavar et al (2012), Bitam et al (2010) used Artificial Bee Colony (ABC) Optimization techniques to find optimal tree solution. Zhufang (2010) used Ant Colony Optimization (ACO) to solve the tree optimization problem. Other heuristic techniques used include Group Search Optimization (GSO) used by Beena \& Sathya (2012) and Co Evolutionary algorithm by Rezaee (2009). Baburaj \& Vasudevan (2008), Nasab et al (2012) proposed particle swarm optimization (PSO) to improve the tree solution. Table 1 discusses the merits and limitations of each technique used in the literature.

From the literature survey it is observed that GA gives better solutions compared to ABC and PSO but convergence time is slow. A good optimization algorithm should achieve globally best solution with fast convergence. To overcome the issues faced with existing optimization techniques various hybrid algorithms have been proposed in the literature involving a global search algorithm with a local search algorithm. To the best of our knowledge hybrid techniques have not been investigated for the multicast routing problem. In this work we propose a new hybrid optimization technique using two popular global optimization techniques GA and PSO.

\section{Problem statement}

A MANET can be represented as a graph $G(N, E)$, where $N=\left\{n_{1}, n_{2}, \ldots, n_{n}\right\}$ is a set of nodes (mobile hosts) and $E=\left\{(i, j) \mid n_{i}, v_{j} \in V\right\}$ a set of links. $(i, j) \in E$ indicates that $n_{i}$ and $n_{j}$ are in control coverage area of each other. Each link $(i, j)$ can be represented by weights which is a combination of delay for data transmission from node $i$ to node $j$ and the distance between the nodes measured using the Euclidean distance. Let $s \in N$ be a multicast source and $D \subseteq N-\{s\}$ a set of destinations. A multicast tree $M(s, D) \subseteq G$ is rooted at $\mathrm{s}$ and reaches all destinations in $D$. The delay of a path on $M$ from $s$ to a destination $n t \in D$, denoted as delay $\left(M\left(s, v_{t}\right)\right)$ becomes the criteria for the routing protocol to find a multicast tree $T^{*}(s, D)$ such that delay is less than or equal to the allowable delay.

\section{Methodology}

This research focuses on a hybrid method combining two heuristic optimization techniques GA and PSO for solving the multicast tree problem. Since GA is encoded using binary values, Binary PSO is used. In Binary PSO, sigmoidal transfer function is used to filp the binary value.

\subsection{Genetic Algorithm}

GA are direct, parallel, stochastic methods for global search/optimization, imitating natural evolution described by Charles Darwin. GA is part of Evolutionary Algorithms (EA) which uses three main principles of natural evolution: reproduction, natural selection and species diversity 


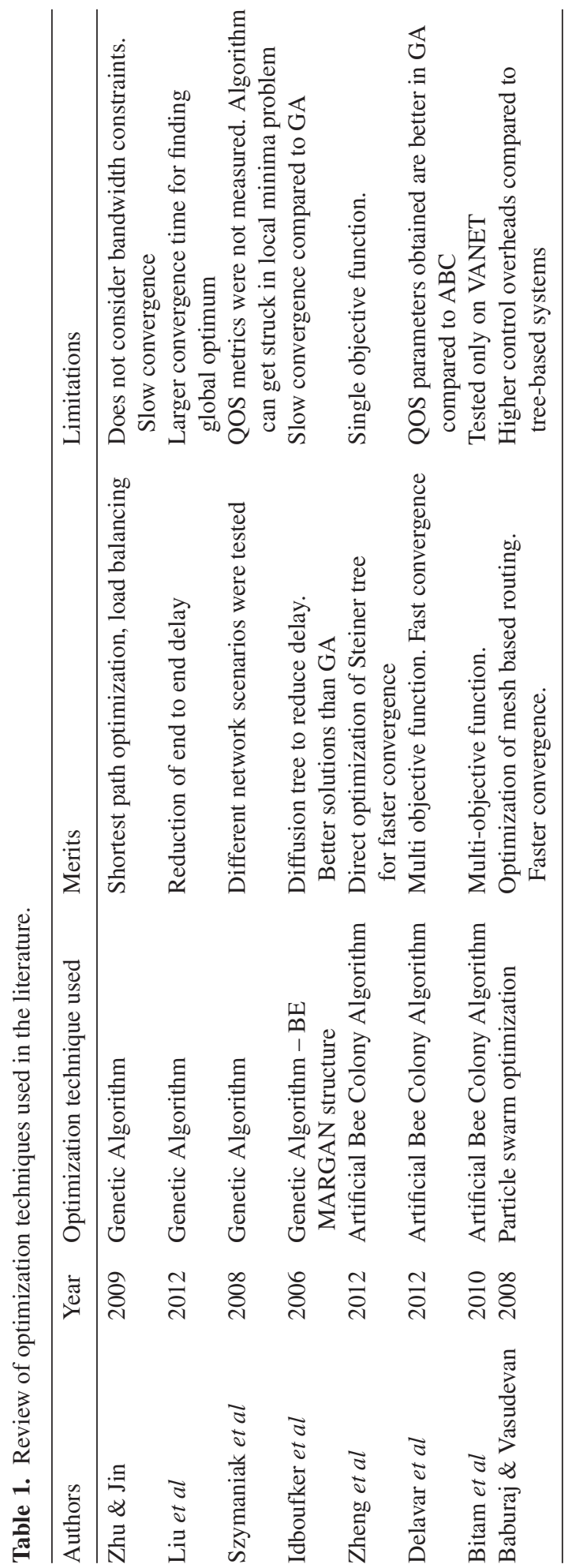


maintained by differences of each generation with the previous one. GA work with an individual's set representing possible task solutions. The selection principle is applied through use of a criterion, evaluating the individual with respect to the solution sought. The best-suited individuals generate the next generation (Popov 2005). The variation operator's role is creation of new individuals from old. Variation operators such as mutation, crossover, parent selection, survivor selection implement elementary steps with search space.

\section{Parent selection mechanism}

The role of parent selection (mating selection) is differentiating among individuals based on quality to permit better individuals to become next generation parents. Parent selection is probabilistic and hence high quality individuals have a higher chance to become parents than those of low quality.

\section{Crossover operator}

Recombination or crossover is a binary variation operator which merges information from two parent genotypes into 1/2 offspring genotypes. Crossover is a stochastic operator and decides the choice of what parts are combined and way of combination. The principle behind crossover is achieved by mating two individuals with different, desirable features producing an offspring combining both features. In this work a two-point crossover operator over selected pair of individuals is implemented.

\section{Mutation operator}

A unitary variation operator is called mutation and applied to one genotype delivering a modified mutant, the child or its offspring. Generally mutation causes a random, unbiased change and has a theoretical role in guaranteeing that search space is well connected.

\section{Survivor selection mechanism}

The role of survivor selection is to differentiate among individuals based on quality. In GA, population size is (most always) constant, hence it has to be decided as to which individuals will be allowed in the next generation. This decision is based on fitness values favouring those with high quality. Survivor selection is often deterministic as opposed to parent selection which is stochastic. For instance, ranking unifies multi-set parents/offspring and selecting top segment (fitness biased), or selection from offspring (age-biased) (Li et al 2010).

The pseudo-algorithm of GA is given in figure 2 .

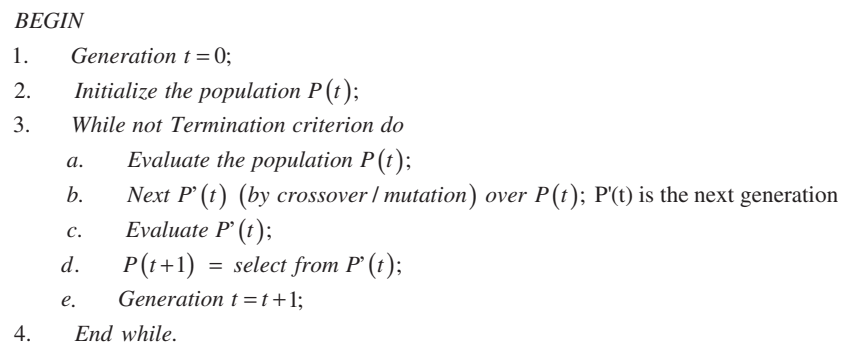

Figure 2. Pseudo-algorithm of GA. 


\subsection{Particle swarm optimization}

The basic PSO components are:

Particle. $\quad X_{i}^{k}$ is a solution $i$ in at iteration $k$ and is the $i^{\text {th }}$ particle of a swarm represented by a $d$-dimensional vector and defined as $X_{i}^{k}=\left[x_{i 1}^{k}, x_{i 2}^{k}, \ldots ., x_{i d}^{k}\right]$, where $x$ 's are optimized parameters and $x_{i d}^{k}$ is position of ith particle in the $d^{\text {th }}$ dimension.

Population. It consists of $\mathrm{n}$ particles in the population and represented by pop $^{k}=\left[X_{1}^{k}, X_{2}^{k}\right.$, $\left.\ldots ., X_{n}^{k}\right]$, where $k$ is the iteration number.

Particle velocity. $\quad V_{i}^{k}$ is particle velocity of particle $i$ at iteration $k$ defined by $V_{i}^{k}=\left[v_{i 1}^{k}, v_{i 2}^{k}\right.$, $\left.\ldots, v_{i d}^{k}\right]$, where $v_{i d}^{k}$ is velocity at $d^{\text {th }}$ dimension.

Particle best. $\quad P B_{i k}$ is best solution of particle $i$ obtained until iteration $k$ and defined by $P B_{i}^{k}=$ $\left[p b_{i 1}^{k}, p b_{i 2}^{k}, \ldots ., p b_{i d}^{k}\right]$ with fitness function $f\left(P B_{i}^{k}\right)$.

Global best. $G B_{k}$ is best position among particles in the swarm and given by $G B^{k}=\left[g b_{1}^{k}\right.$, $\left.g b_{2}^{k}, \ldots, g b_{d}^{k}\right]$ with fitness function $f\left(G B^{k}\right)$.

Termination criterion. It is the condition where the search process is terminated.

In BPSO dimension a binary value of 0 or 1 is assigned with a probability of 0.5 . In particular,

$$
\begin{gathered}
\text { if } U(0,1)>0.5, \text { then } x_{i d}^{0}=1 \\
\text { else } x_{i d}^{0}=0 .
\end{gathered}
$$

To avoid infeasible solutions the velocity values are restricted by $v_{i}^{k}=\left[v_{\min }, v_{\max }\right]=[-6,6]$ where $V_{\min }=-V_{\max }$. Velocity of particle $i$ in $d^{\text {th }}$ dimension is established by $v_{i d}^{0}=v_{\min }+$ $\left(v_{\max }-v_{\min }\right) * \operatorname{rand}()$. This limit enhances local search exploration of problem space.

As binary version of PSO algorithm is used, a transfer function for generating new solutions and force the real values between 0 and 1 is required. When a velocity value is computed, the velocity range is first restricted to closed interval $\left\lfloor V_{\min }, V_{\max }\right\rfloor$ as shown in the following equation.

$$
h\left(v_{i d}^{k}\right)= \begin{cases}V_{\max }, & \text { if } v_{i d}^{k}>V_{\max } \\ v_{i d}^{k}, & \text { if }\left|v_{i d}^{k}\right| \leq V_{\max } \\ V_{\min }, & \text { if } v_{i d}^{k}<V_{\min } .\end{cases}
$$

A sigmoid function is then used to scale velocities between 0 and 1 and shown in the following equation

$$
\operatorname{sigmoid}\left(v_{i d}^{k}\right)=\frac{1}{1+e^{-v_{i d}^{k}}} .
$$

New solutions are found by updating velocity and dimension respectively. To do that first, change in velocity $v_{i d}^{k}$ is computed so that

$$
\Delta v_{i d}^{k-1}=c_{1} r_{1}\left(p b_{i d}^{k-1}-x_{i d}^{k-1}\right)+c_{2} r_{2}\left(g b_{d}^{k-1}-x_{i d}^{k-1}\right) .
$$


Velocity $v_{i d}^{k}$ is then update by using the following equation

$$
v_{i d}^{k}=h\left(v_{i d}^{k-1}+\Delta v_{i d}^{k-1}\right) .
$$

Finally dimension $d$ of particle $i$ is updated such that

$$
x_{i d}^{k}=\left\{\begin{array}{lr}
1, & \text { if } U(0,1)<\operatorname{sigmoid}\left(v_{i d}^{k}\right) . \\
0, & \text { otherwise }
\end{array}\right.
$$

\subsection{Solution representation}

For a source $s$ connected to $v_{\mathrm{i}}$ destinations, the chromosome can be represented by genes where each gene represents a route $j$ for destination $v_{\mathrm{i}}$ from source $s$. In Binary PSO this represents an initial particle. For example, figure 3 shows a six-node network with node reachability shown by thick lines.

The solution representation for S1 to D1 are

$$
\begin{aligned}
& 001 \rightarrow S 1-V 5-D 1 \\
& 010 \rightarrow S 1-V 1-V 3-D 1 \\
& 011 \rightarrow S 1-V 5-V 1-V 3-D 1
\end{aligned}
$$

Similarly representation for $\mathrm{S} 1$ to $\mathrm{D} 2$ are

$$
\begin{aligned}
& 001 \rightarrow S 1-V 1-D 2 \\
& 010 \rightarrow S 1-V 5-V 1-D 2
\end{aligned}
$$

The solution generated are evaluated using the proposed fitness function.

\subsection{Proposed fitness function}

The fitness function at instance $t$ is based on the jitter and packet delivery ratio (PDR) which is crucial for multicast communication and is given by Eq. (6). Since node acts as router

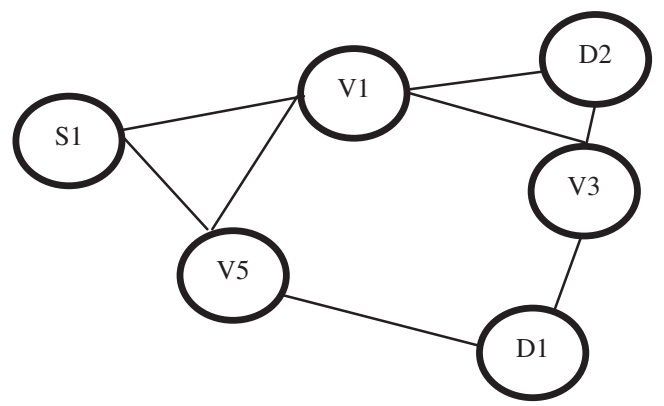

Figure 3. Solution representation. 
also, the data generated and transmitted from the node forms the sum of packets handled by the node.

$$
\begin{aligned}
F(t) & =\sqrt{\frac{2 J_{m}}{\lambda_{\text {out }}} e^{-\frac{\lambda_{g}^{2}}{2 \lambda_{\text {in }}} J_{m}} P D R} \\
J_{m} & =\max \text { jitter, } \lambda_{\text {in }}=\text { input_packet_to_node } \\
\lambda_{g} & =\text { Generated_packet_in_node, } \lambda_{\text {out }}=\lambda_{\text {in }}+\lambda_{g}, \\
P D R & =\text { Packet_delivery_ratio }
\end{aligned}
$$

\subsection{Proposed GA-PSO}

The flow chart of the proposed hybrid algorithm is shown in figure 4.

In the proposed system the initial solutions are first selected using Roulette wheel selection. This ensures sub-optimal solutions with very large number of hops are eliminated. The selected solutions become the input for the Binary PSO which computes the new solutions after computation of velocity. A reward-based selection algorithm is used to eliminate suboptimal solutions which are then used in the next iteration. The selected solutions act as parents and the next generation of solutions is created using crossover and mutation. The solutions are ranked and if the best solution meets the termination criteria then solution is terminated or the algorithm proceeds to the next iteration.

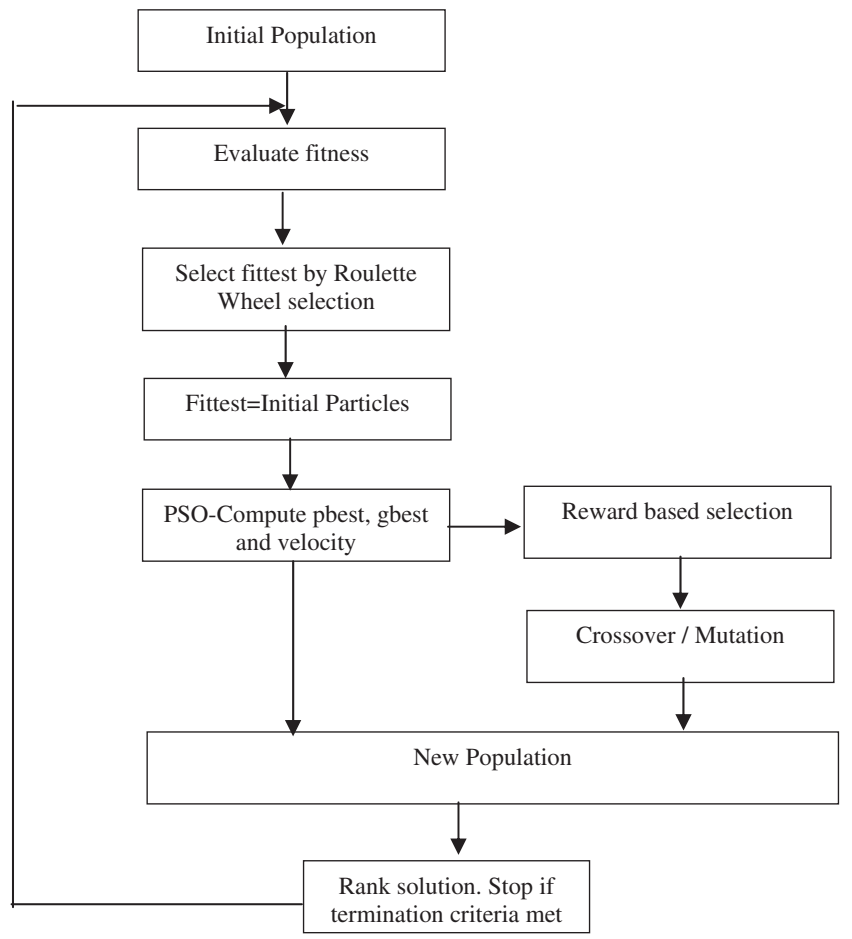

Figure 4. Flow chart of the proposed hybrid algorithm. 


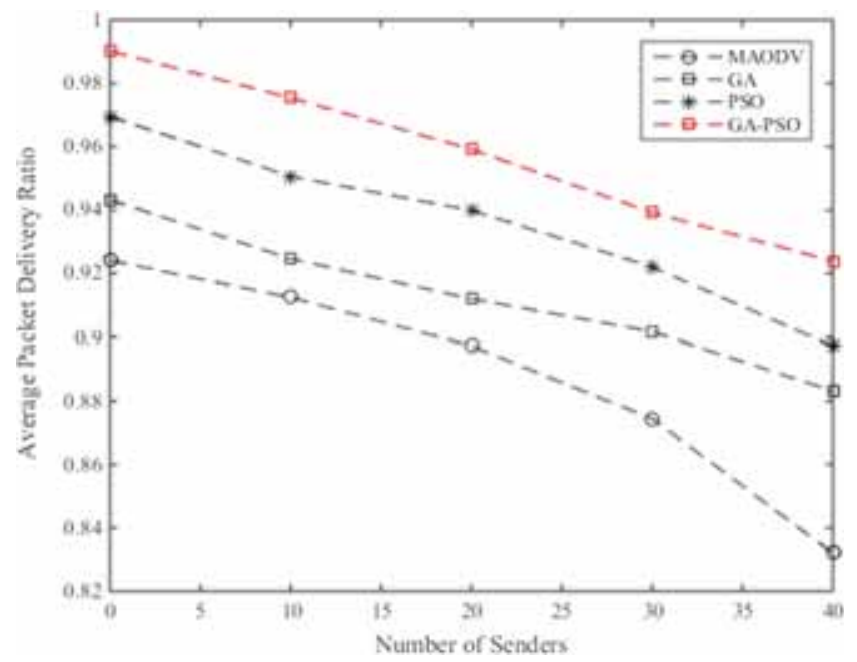

Figure 5. Average Packet delivery ratio.

\section{Experiments and results}

Simulations were carried out in a 3,000 sq.m network consisting of 100 nodes. The transmission range of each node is $220 \mathrm{~m}$ using an Omni directional antenna. The number of senders was varied from 1 to 40 in increments of 10 and random way point mobility model was used with a maximum node speed of $30 \mathrm{kmph}$. Simulations were carried out using MAODV, GAbased method, PSO-based method and proposed GAPSO method. Figure 5 shows the PDR under different scenarios. The proposed GA-PSO outperforms all other techniques with an average improvement of $7.53 \%$ compared with MAODV and about $2.25 \%$ improvement compared to other techniques.

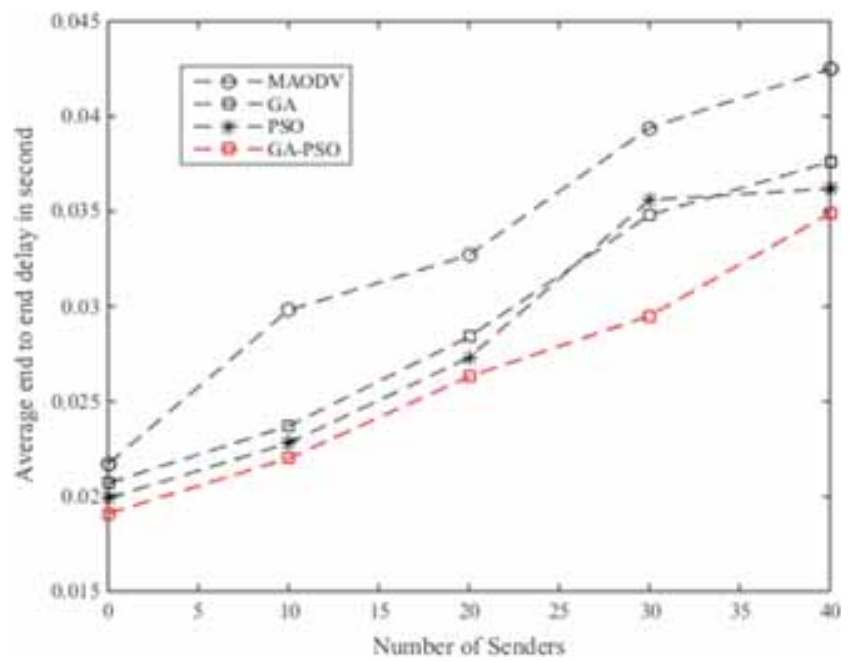

Figure 6. Average end to end delay. 


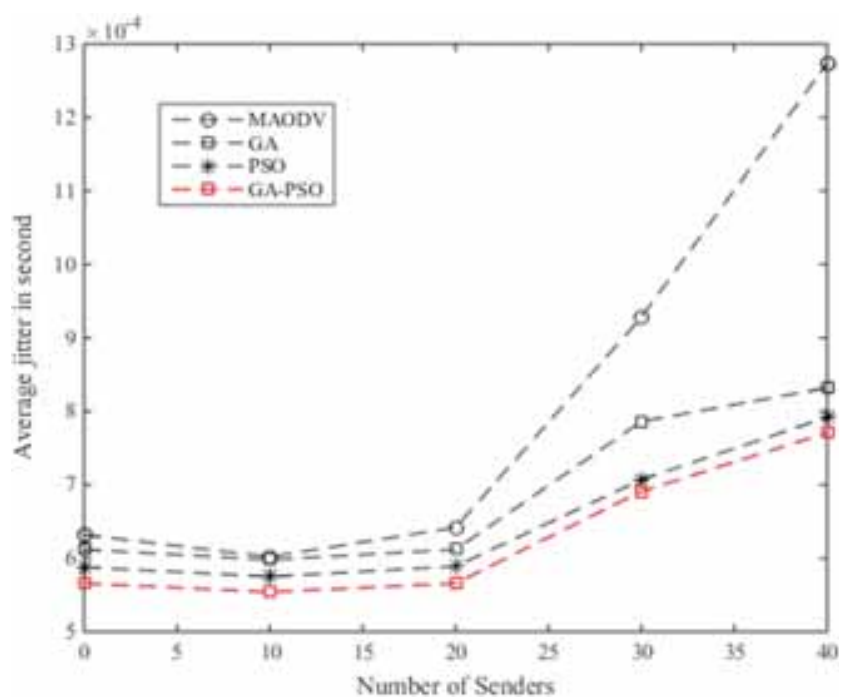

Figure 7. Average jitter in seconds.

It is also observed that performance of MAODV starts deteriorating as the number of senders is increased. Figure 6 shows the end to end delay obtained across different scenarios. GA-based solution and PSO-based solution have similar end to end delays and statistically the difference may be negligible. The average end to end delay of the proposed system is lower by about $7.6 \%$ compared to GA and PSO-based method and by $23.03 \%$ compared to MAODV.

Figure 7 shows average jitter measured in seconds where again the inter packet arrival time for MAODV is high compared to other techniques. The higher jitter is due to the increased number of hops and packet drops as the number of senders increase. Jitter may not be critical for most applications except for multimedia applications. The plot of cost function is shown in figure 8 .

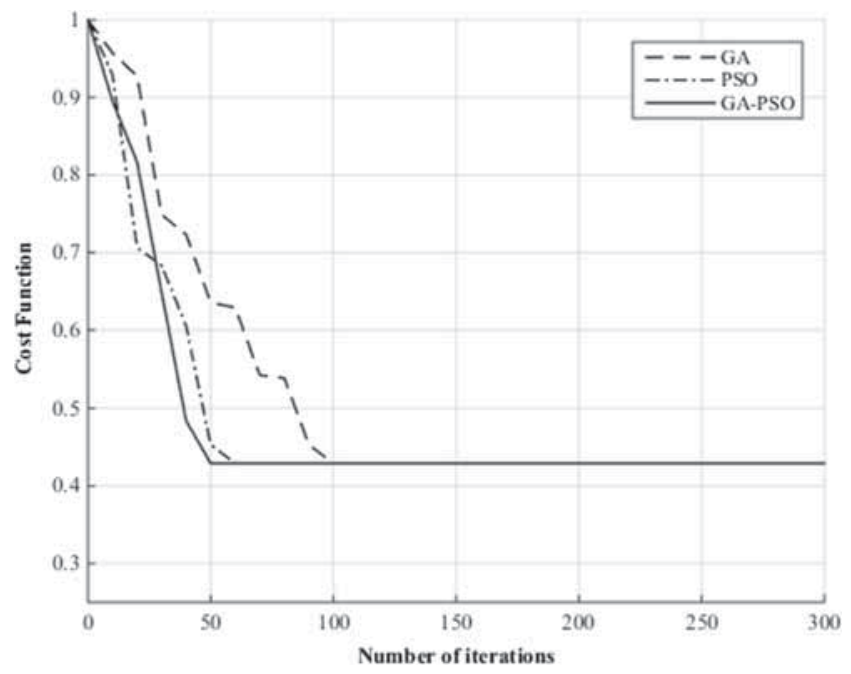

Figure 8. Cost function. 
As seen in the literature survey the convergence time of GA is higher than other two techniques with the convergence of PSO and GAPSO being almost the same. The cost function value between all the three techniques is in the standard deviation of about $3.2 \%$ which is seen in the QOS parameters.

\section{Conclusion}

Multicasting is a challenging task in MANETs because of high node mobility and frequent topology change. Multicasting is one of the alternatives of multiple unicast transmissions when same data has to be transmitted for a group of nodes. This work proposed a hybrid optimization based on GA and PSO for selecting optimal routes to group nodes for multicasting. Simulations were conducted by varying number of mobile nodes, and results compared with multicast AODV (MAODV) protocol using jitter, end to end delay and PDR as QOS parameters. Results show that PDR is improved, delay is decreased and jitter is reduced in the proposed hybrid method when compared to MAODV, GA and PSO based techniques. Further investigations can be carried out on the impact of node pause time in the proposed algorithm.

\section{References}

Baburaj E and Vasudevan V 2008 An intelligent mesh based multicast routing algorithm for MANETs using particle swarm optimization. Int. J. Comput. Sci. Netw. Security 8(5): 214-218

Beena and Sathya M 2012 A multi-objective optimization strategy based on GSO for the multicast routing problem. Int. J. Adv. Res. Comput. Sci. Softw. Eng. 2(10): 326-333

Biswas J, Barai M and Nandy S K 2004 Efficient hybrid multicast routing protocol for ad-hoc wireless networks. In: 29th Annual IEEE International Conference on Local Computer Networks, 2004. pp. 180187

Bitam S, Batouche M and Talbi E G 2010 A survey on bee colony algorithms. In: 2010 IEEE International Symposium on Parallel and Distributed Processing, Workshops and Phd Forum (IPDPSW), pp. 1-8

Cheng H and Yang S 2010 Genetic algorithms with immigrants schemes for dynamic multicast problems in mobile ad hoc networks. Eng. Appl. Artif. Intell. 23(5): 806-819

Delavar A G, Hoseyny S and Maghsoudi R 2012 BCO-based optimized heuristic strategies for QoS routing. J. Math. Comput. Sci. 5(1): 105-114

Idboufker N, Juidette H and Berraissoul A 2006 An efficiency based genetic approach for multicast best effort routing. In: Proceedings ISCCSP Second International Symposium on Communications, Control and Signal processing

Ji L and Corson M S 2001 Differential destination multicast-a manet multicast routing protocol for small groups. In: INFOCOM 2001. Twentieth Annual Joint Conference of the IEEE Computer and Communications Societies. Proceedings. IEEE vol. 2, pp. 1192-1201

Kunz T and Cheng E 2001 Multicasting in ad-hoc networks: Comparing MAODV and ODMRP. In: Proceedings of the Workshop on Ad hoc Communications, pp. 186-190. Bonn, Germany

Lee S J, Su W and Gerla M 2002 On-demand multicast routing protocol in multihop wireless mobile networks. Mobile Netw. Appl. 7(6): 441-453

Li Z, Liu X, Duan X and Huang F 2010 Comparative research on particle swarm optimization and genetic algorithm. Comput. Inform. Sci. 3(1): P120

Liu K, Chen Z, Abraham A, Cao W and Jing S 2012 Degree-constrained minimum spanning tree problem using genetic algorithm. In: 2012 Fourth World Congress on Nature and Biologically Inspired Computing (NaBIC), pp 8-14

Nasab A S, Derhami V, Khanli L M and Bidoki A M Z 2012 Energy-aware multicast routing in manet based on particle swarm optimization. Proced. Technol. 1: 434-438 
Popov A 2005 Genetic algorithms for optimization. User Manual, Hamburg

Rezaee A 2009 Using of coevolutionary algorithm on P2P networks. Serbian J. Elect. Eng. 6(1): 43-55

Szymaniak P, Piechowiak M and Zwierzykowski P 2008 New genetic multicast routing algorithm for ad-hoc networks. Image Process. Commun. 13(1-2): 3-11

Viswanath K, Obraczka K and Tsudik G 2006 Exploring mesh and tree-based multicast. Routing protocols for MANETs. IEEE Trans. Mobile Comput. 5(1): 28-42

Wu C W and Tay Y C 1999 AMRIS: A multicast protocol for ad hoc wireless networks. In: Military Communications Conference Proceedings, 1999. MILCOM 1999. IEEE, vol. 1, pp. 25-29

Xie J, Talpade R R, Mcauley A and Liu M 2002 AMRoute: Ad hoc multicast routing protocol. Mobile Netw. Appl. 7(6): 429-439

Yi Y, Lee S J, Su W and Gerla M 2003 On-demand multicast routing protocol (ODMRP) for ad hoc networks. draft-yi-manet-odmrp-00. txt

Zheng Z, Wang H and Yao L 2012 An Artificial Bee Colony Optimization algorithm for multicast routing. In: 2012 14th International Conference on Advanced Communication Technology (ICACT). pp. 168-172

Zhu C and Jin M 2009 An anycast routing algorithm based on genetic algorithm. WSEAS Trans. Comput. 8(1): 113-122

Zhufang K 2010 An multicast routing based on ant colony optimization algorithm for DTN. In: 2010 Fourth International Conference on Genetic and Evolutionary Computing (ICGEC). pp. 354-357 